\title{
Primera temporada de nombramiento de borrascas en AEMET (2017-2018)
}

\author{
Jaime Rey Vidaurrázaga ${ }^{1}$ (jreyv@aemet.es) \\ Francisco Javier Rodríguez Marcos ${ }^{1}$ (frodriguezm@aemet.es) \\ Rubén del Campo Hernández ${ }^{2}$ (rcampoh@aemet.es)
}

${ }^{1}$ AEMET / Departamento de Producción

${ }^{2}$ AEMET / Área de Información Meteorológica y Climatológica

\begin{abstract}
RESUMEN
El primer esquema sistematizado para nombrar ciclones comenzó a funcionar en el año 1953 con el objetivo de ayudar a la identificación rápida de ciclones tropicales en los mensajes transmitidos por estaciones de radio, bases costeras y buques de guerra, al ser los nombres más sencillos de recordar que los números y términos técnicos. Desde entonces, en Europa han ido surgiendo distintas iniciativas a nivel nacional o regional para nombrar los distintos ciclones o anticiclones extratropicales, lo que ha llevado a que en los últimos años una misma borrasca pueda ser nombrada de múltiples maneras por distintos organismos. Para evitar la confusión asociada y para potenciar la voz única de los Servicios Meteorológicos e Hidrológicos Nacionales (SMHN), en el año 2013 se creó un grupo de trabajo dentro del WGCEF (Working Group On Cooperation between European Forecasters) de EUMETNET.
\end{abstract}

En la temporada 2015-16, Met Office y Met Eireann, que formaron el Grupo Oeste, empezaron a nombrar las borrascas que producían gran impacto, basándose en la emisión de avisos naranjas de viento basados en impactos. El objetivo del grupo de trabajo del WGCEF era crear diferentes grupos regionales para posteriormente unirse y conseguir un sistema único a nivel europeo. Por ello, de cara a la temporada 2017-2018 se creó el Grupo Suroeste, en el que AEMET, junto con IPMA(Instituto Portugués del Mar y la Atmósfera) y Météo-France, empezó a nombrar de forma operativa las borrascas atlánticas en diciembre de 2017.

En este trabajo se analiza el efecto en la difusión de la información meteorológica del nombramiento de las borrascas, analizando los accesos a la página web de AEMET y la difusión a través de redes sociales de la información emitida por la Agencia, en una temporada que ha sido especialmente activa en nuestras latitudes debido a un patrón de NAO negativa.

PALABRAS CLAVE: storm naming; nombramiento de borrascas; ciclón extratropical; borrasca; aviso; viento; impactos; EUMETNET.

\section{INTRODUCCIÓN}

En un comienzo, la práctica de nombrar ciclones se realizó con el fin de ayudar a identificar rápidamente los ciclones y facilitar la difusión de mensajes meteorológicos entre estaciones de radio, bases costeras y buques de guerra, debido a que se presume que los nombres son mucho más fáciles de recordar que los números y 
los términos técnicos. Este nombramiento se empezó a realizar de una manera arbitraria. Por ejemplo, un ciclón en el Atlántico que arrancó el mástil de un barco llamado Antje pasó a llamarse huracán Antje. Tiempo después, a mediados de 1900, se inició la práctica de utilizar nombres femeninos para los ciclones. [1]

Posteriormente, en la búsqueda de un sistema de nombres más organizado y eficiente, los meteorólogos decidieron identificar los ciclones utilizando nombres de una lista organizada de forma alfabética. Por lo tanto, una tormenta con un nombre que comienza con A, como Anne, sería la primera tormenta en ocurrir en el año.

Desde 1953, los ciclones tropicales del Atlántico han sido nombrados a partir de listas originadas por el Centro Nacional de Huracanes de Estados Unidos. En la actualidad, estas listas son mantenidas y actualizadas por un comité internacional de la Organización Meteorológica Mundial. Las listas de nombres originales solo incluían nombres de mujeres, pero desde 1979 se alternan los nombres masculinos y femeninos.

En este periodo, en Europa han ido surgiendo distintas iniciativas a nivel nacional o regional para nombrar los distintos ciclones o anticiclones extratropicales. Entre estas iniciativas destaca la de la Universidad Libre de Berlín [2], que permite «bautizar» centros de altas y de bajas presiones a cambio de una aportación económica destinada a financiar proyectos científicos de dicha Universidad. Esta situación ha provocado que en ocasiones se denominara a una misma borrasca con distintos nombres, generando confusión. Por ello, en el año 2013, surgió una iniciativa por parte de EUMETNET, dentro del Working Group on Cooperation between European Forecasters (WGCEF), con el objetivo de que cada borrasca con gran impacto tuviera un único nombre (CuSACK et al., 2017). Es muy probable que en un futuro esta actividad se convierta en una actividad financiada dentro del Programa de Predicción para la siguiente fase de EUMETNET (2019-23), llamada Storm naming.

El propósito de Storm naming es definir un esquema único a nivel europeo en el que participen todos los Servicios Meteorológicos e Hidrológicos Nacionales (SMHN). El nombre asignado a cada borrasca será el usado tanto por los propios SMHN como por los medios de comunicación de cada país. El objetivo final es la integración del esquema de Storm naming en Europa, probablemente con la existencia de una lista única de nombres.

Los principales beneficios del nombramiento de borrascas con gran impacto son los siguientes:

a) voz autorizada única de los SMHN;

b) nombre único, evitando confusiones con los distintos nombres que podrían darse por distintos SMHN y medios de comunicación;

c) comunicación sencilla;

d) oportunidad de colaboración entre los SMHN en predicción operativa;

e) facilidad para su uso posterior (ej. casos de estudio).

En la actualidad hay varios grupos regionales en distintos niveles de operatividad que hay que terminar de completar, cada uno con una lista propia, que son:

- Oeste (W; Irlanda y Reino Unido): lleva ya tres campañas en funcionamiento.

- Suroeste (SW; Portugal, Francia y España): lleva una campaña en funcionamiento.

- Otros: Norte (Noruega, Suecia, Dinamarca, Finlandia), Central (Alemania, Austria, Suiza, Luxemburgo, Eslovaquia, Hungría, Polonia), etc. 


\section{CREACIÓN DEL GRUPO SUROESTE Y PRIMERA TEMPORADA DE NOMBRAMIENTO DE BORRASCAS}

En el año 2017, los servicios meteorológicos de Francia (Météo-France), Portugal (IPMA) y España (AEMET) llegaron a un acuerdo para nombrar las borrascas atlánticas con mayor impacto sobre su ámbito de predicción, de manera coordinada con el grupo Oeste. El acuerdo, con fecha de inicio operativo el 1 de diciembre de 2017, se alcanzó tras definir una serie de criterios comunes, detallados en el apartado 2.1. A lo largo de esta primera temporada, se nombraron un total de nueve borrascas, cuatro por AEMET, tres por el IPMA y dos por Météo-France, como se detalla en el apartado 2.2.

\subsection{Criterios de nombramiento de borrascas en el Grupo Suroeste para la temporada 2017-2018}

a) Que se prevean condiciones que den lugar a la emisión de avisos de viento (en el caso de AEMET, racha máxima) de nivel naranja o rojo, lo más orientados posibles a impactos, asociados al paso de una borrasca.

b) Los avisos asociados a vientos locales o regionales (cierzo, tramontana, levante del Estrecho, etc.) no se tendrán en cuenta.

c) Área de formación: océano Atlántico. Para la temporada 2017-2018 quedaron excluidas las borrascas formadas en el Mediterráneo occidental.

d) Las borrascas conservarán el mismo nombre durante todo el periodo de su vida activa.

e) Si una borrasca ya nombrada por otro grupo se dirige a nuestras zonas, se debe respetar y conservar el mismo nombre dado por el otro grupo (coordinación entre Met Office y Météo-France).

f) En el caso de un ciclón post-tropical, se deberá usar el mismo nombre dado por el Centro Nacional de Huracanes $(\mathrm{CNH})$ de Miami, incluyendo el prefijo «ex-».

\subsection{Borrascas nombradas durante la temporada 2017-2018}

En la tabla 1 se presenta la información más relevante de las distintas borrascas nombradas. Como se puede ver, los tres servicios meteorológicos implicados nombraron alguna borrasca. Además, y es importante tenerlo en cuenta, el hecho de que una borrasca sea nombrada no implica que su impacto tenga que alcanzar a todos los países implicados, sino que basta con que afecte a alguna zona del conjunto. Dos ejemplos claros son David, que afectó principalmente al norte de Francia (aunque posteriormente provocó tiempo adverso en Bélgica, Países Bajos y Alemania), e Irene, cuyos impactos afectaron exclusivamente a las islas Azores y a áreas de alta mar.

\begin{tabular}{|c|c|c|c|}
\hline $\begin{array}{c}\text { Nombre de } \\
\text { la borrasca }\end{array}$ & $\begin{array}{c}\text { Servicio Meteorológico } \\
\text { Nacional }\end{array}$ & $\begin{array}{c}\text { Fecha de } \\
\text { nombramiento }\end{array}$ & $\begin{array}{c}\text { Fecha de impactos } \\
\text { sobre España }\end{array}$ \\
\hline Ana & AEMET & 8 diciembre 2017 & $10-11$ diciembre 2017 \\
\hline Bruno & AEMET & 25 diciembre 2017 & $26-27$ diciembre 2017 \\
\hline Carmen & Météo-France & 31 diciembre 2017 & 31 diciembre 2017 \\
\hline David & Météo-France & 17 enero 2018 & - \\
\hline Emma & IPMA & 25 febrero 2018 & 28 febrero - 2 marzo 2018 \\
\hline Félix & IPMA & 8 marzo 2018 & 10 -11 marzo 2018 \\
\hline Gisele & AEMET & 12 marzo 2018 & 14 marzo 2018 \\
\hline Hugo & AEMET & 22 marzo 2018 & $23-24$ marzo 2018 \\
\hline Irene & IPMA & 16 abril 2018 & - \\
\hline
\end{tabular}

Tabla 1. Borrascas nombradas por el Grupo SW durante la temporada 2017-18. 


\section{ANÁLISIS DEL EFECTO EN LA DIFUSIÓN DE LA INFORMACIÓN METEOROLÓGICA DEL NOMBRAMIENTO DE BORRASCAS}

Se ha optado por analizar el alcance de la difusión del nombramiento de borrascas en los dos medios que utiliza AEMET y que cuentan con mayor repercusión: la página web, que sobrepasa los cuatro millones de visitas diarias, y la red social Twitter, donde la cuenta principal de AEMET cuenta con más de 175000 seguidores.

\subsection{Impacto en las visualizaciones de la web de AEMET}

Para analizar el efecto del nombramiento de las borrascas en la visualización de la web de AEMET se ha optado por trabajar con los datos de los tres últimos años, concretamente en el periodo comprendido entre el 22 de enero de 2016 (se han descartado los datos de principios de año por cambios en el conteo del número de visitas a la web) y el 31 de mayo de 2018 (fecha posterior a la última borrasca nombrada y cercana a la celebración del Simposio).

Como se observa en la figura 1 existe una relación directa entre el número de visitas a la web y las borrascas nombradas con un impacto significativo sobre España. Esto era esperable, ya que prácticamente todos los picos en el número de visitas web están asociados, o bien a situaciones de tiempo adverso, o bien a periodos asociados a fiestas nacionales. Pero también hubo episodios con un gran número de visitas en la web en los que no se nombró a ninguna borrasca, que en general coincidieron con nevadas copiosas en amplias áreas y con la emisión de avisos naranjas de dicho fenómeno. De hecho, el 5 de febrero de 2018 se batió el récord de visitas a la web desde que se tienen registros, que coincidió con una nevada de unos $5 \mathrm{~cm}$ en el área metropolitana de Madrid, con el correspondiente alto impacto.

Por otro lado, el nombramiento de borrascas sin impacto sobre España no parece tener efecto sobre el número de visitas a la web.

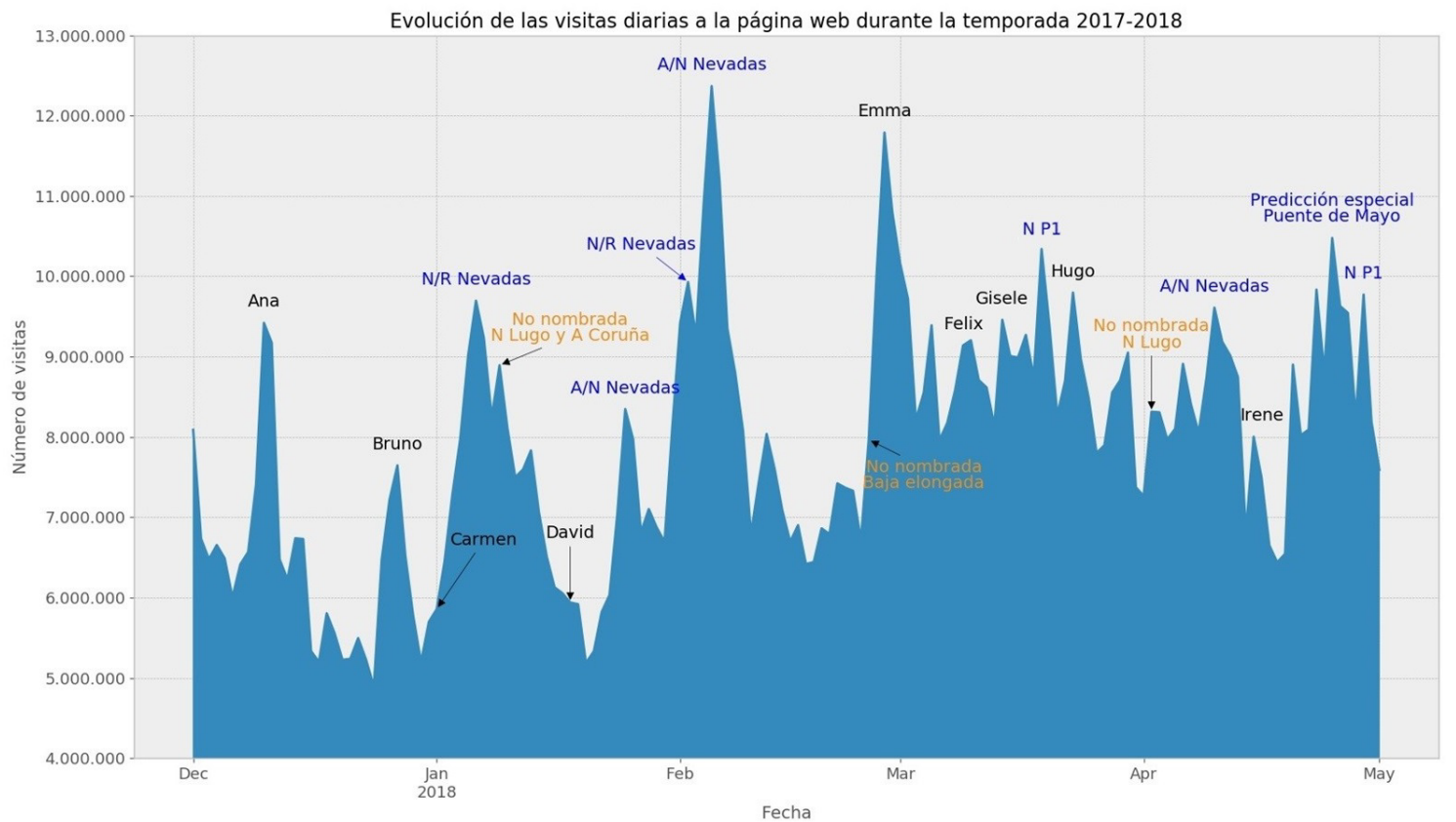

Figura 1. Páginas totales visitadas en la web de AEMET entre el 1 de diciembre de 2017 y el 30 de abril de 2018. Se resaltan las distintas borrascas nombradas, las que no se nombraron, pero generaron dudas sobre su posible nombramiento, así como los episodios que generaron los mayores picos en los accesos a la web. 


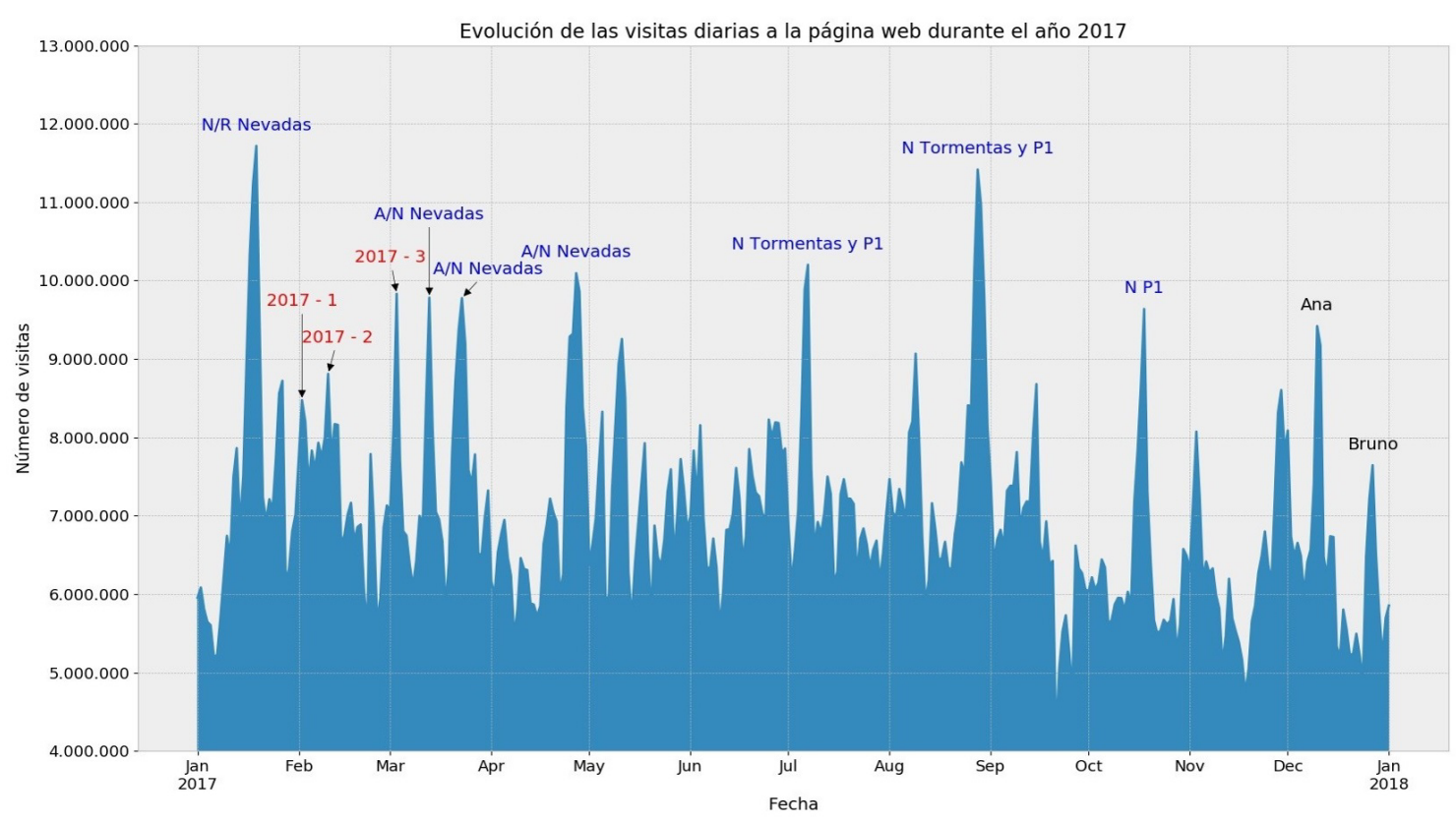

Figura 2. Páginas totales visitadas en la web de AEMET entre el 1 de enero de 2017 y el 31 de diciembre de 2017. Se resaltan las distintas borrascas nombradas, las que se habrían nombrado en la temporada anterior, así como los episodios que generaron los mayores picos en los accesos a la web.

Del análisis de la figura 2, correspondiente al año 2017, también se pueden sacar conclusiones interesantes. Entre enero y abril se produjeron tres borrascas que habrían sido nombradas en caso de haber estado implementado en esa época el nombramiento de borrascas en el grupo SW. Sin embargo, lo llamativo es que los picos más significativos en dicho periodo corresponden a episodios de nevadas significativas y de precipitaciones y tormentas intensas con avisos naranjas asociados, en los que, de existir una borrasca (que no siempre la hubo), esta no cumplió los criterios establecidos para el nombramiento.

En la figura 3 se puede ver que, durante el año 2016, el día con mayor número de visitas a la web tuvo relación con una situación meteorológica que habría dado lugar al nombramiento de una borrasca.

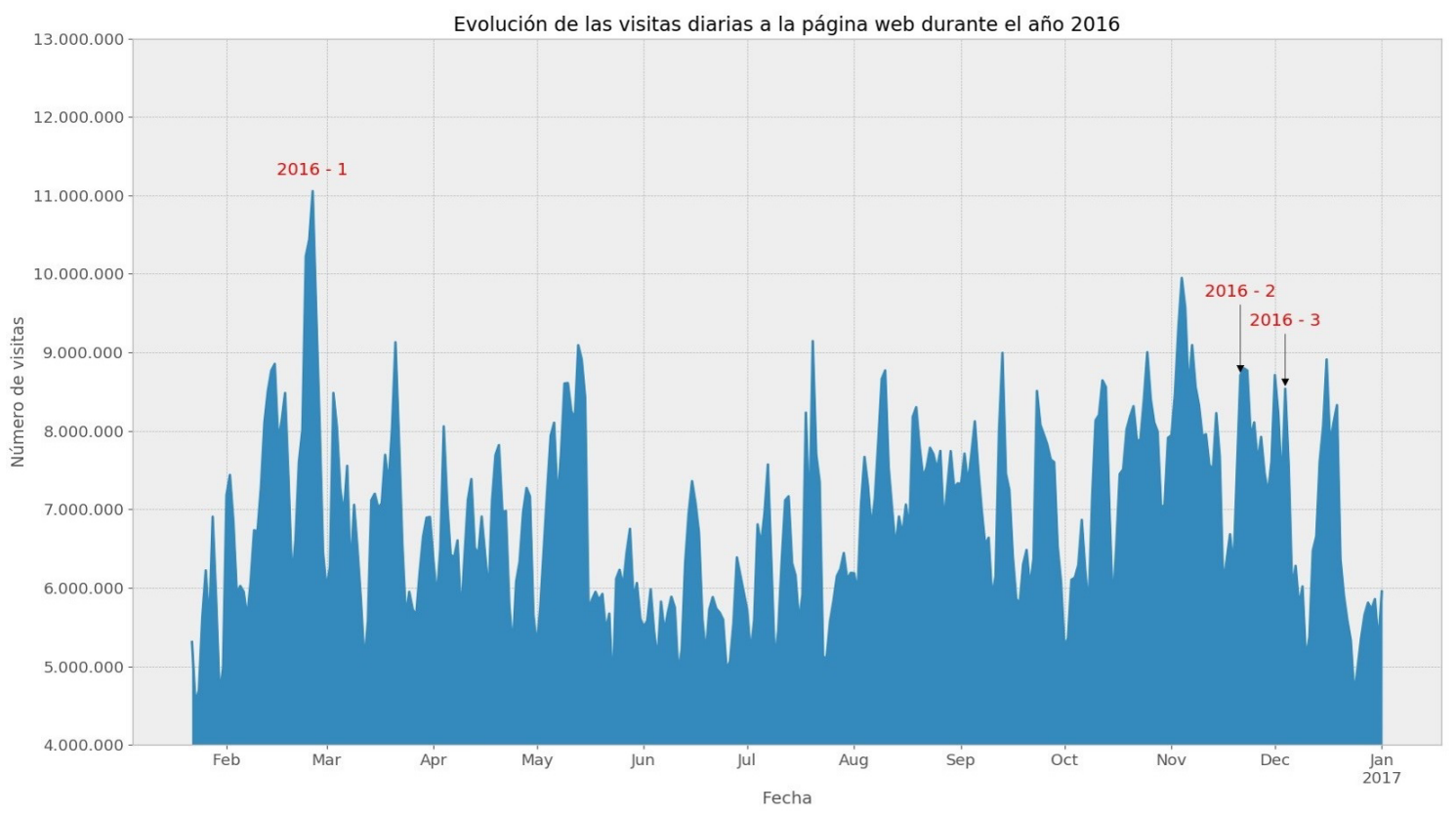

Figura 3. Páginas totales visitadas en la web de AEMET entre el 22 de enero de 2016 y el 31 de diciembre de 2016. Se resaltan las distintas borrascas que se habrían nombrado en dicho periodo. 
De todo lo anterior se puede concluir que, si bien el nombramiento de borrascas está relacionado con máximos en el número de visitas web, no hay evidencias de que su nombramiento produzca un incremento claro en el número de visitas. Además, sería interesante analizar las situaciones de nevadas significativas referidas, pues generan un gran número de visitas web, pudiendo estar asociadas a borrascas que no se nombran por no llevar asociados avisos naranjas de viento, aunque en algunos de estos casos las bajas de niveles medios y altos no tienen prácticamente reflejo en superficie.

\subsection{Impacto en el seguimiento de la cuenta principal de AEMET en la red social Twitter (@AEMET_Esp)}

En el análisis del impacto del nombramiento de borrascas en la red social Twitter se utilizan términos muy relacionados con el análisis del alcance que tienen los tuits individuales dentro de la red social. Por ello, es conveniente definirlos previamente:

- Impresiones: número de veces que se muestra el contenido de un tuit.

- Interacciones: número de veces que se hace clic en un tuit o en su contenido.

Con el fin de facilitar su seguimiento y mejorar su difusión cada borrasca contó con una etiqueta del tipo \#BorrascaNombre.

Como se observa en la figura 4 también existe una relación directa entre el número de impresiones de los tuits de un día y las borrascas nombradas con un impacto significativo sobre España. Además, se observa que todas las borrascas han tenido una gran difusión, incluso cuando no tuvieron impacto sobre nuestro territorio. En la figura 5 se puede ver que tanto los tuits destacados de cada borrasca, como la media de impresiones de cada tuit con la etiqueta \#BorrascaNombre correspondiente alcanzaron una clara mayor difusión que la media de todos los tuits del mes. Además, es muy destacable la gran difusión que tuvo el tuit destacado de la borrasca Ana y la gran aceptación que tuvo la implantación del sistema de nombramiento de borrascas. En la tabla 2, donde se representan distintos marcadores de la difusión de los tuits, se puede ver cómo, una vez superado el periodo inicial de máximo interés en la red social del nombramiento de borrascas, la difusión de las borrascas que produjeron una mayor afección en nuestro país se ha mantenido en unos números notables (más de 60000 impresiones y 3000 interacciones por borrasca).

Finalmente, como curiosidad, cabría resaltar que desde una cuenta oficial de una protección civil autonómica se le dio nombre a la borrasca Hugo antes de que fuera nombrada oficialmente por AEMET, por lo que tuvo que ser desmentido por la Agencia. En todo caso, se trató de un hecho puntual.

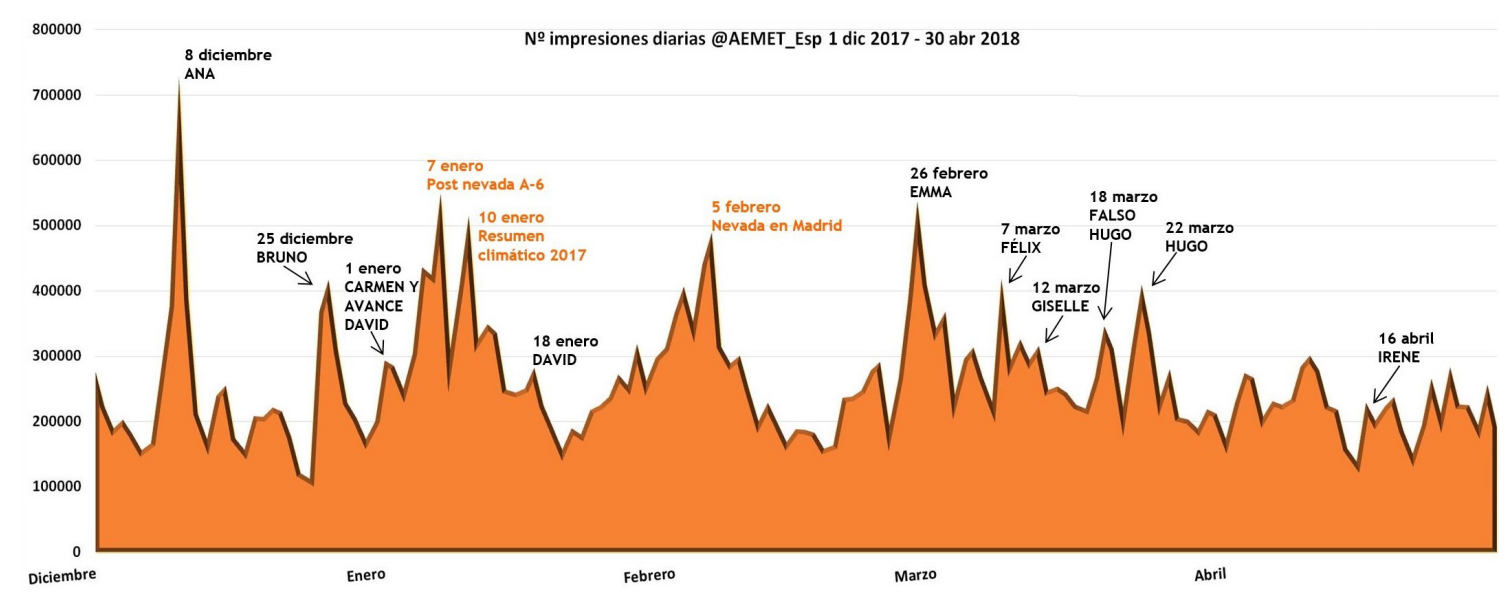

Figura 4. Impresiones diarias en la cuenta de Twitter @ AEMET_Esp entre el 1 de diciembre de 2017 y el 30 de abril de 2018. Se resaltan las distintas borrascas nombradas, así como los episodios que generaron los mayores picos en los accesos a la web. 


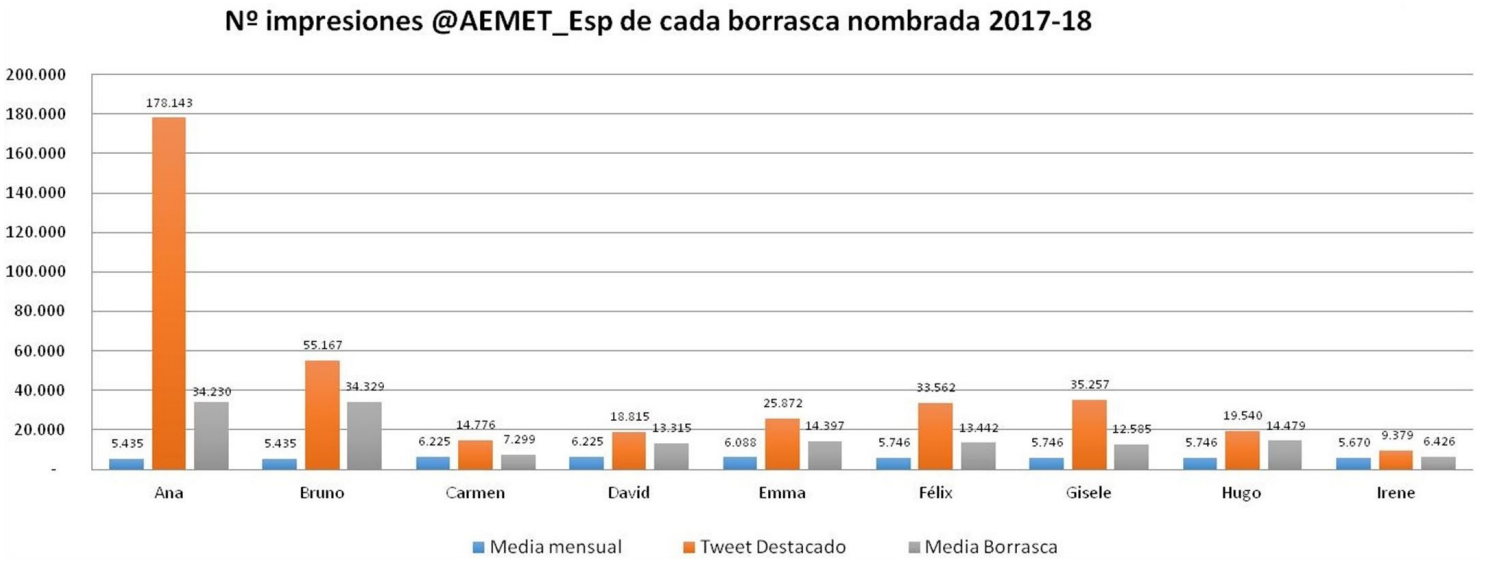

Figura 5. Comparación entre impresiones medias mensuales, impresiones del tuit más destacado de cada borrasca, y la media de las impresiones de los tuits de las borrascas.

\begin{tabular}{|c|c|c|c|c|}
\hline Borrasca & $\begin{array}{c}\text { Impresiones } \\
\text { totales }\end{array}$ & $\begin{array}{c}\text { Interacciones } \\
\text { totales }\end{array}$ & Me gusta & Retuits \\
\hline Ana & 479226 & 28108 & 1098 & 1083 \\
\hline Bruno & 205973 & 10029 & 392 & 345 \\
\hline Carmen & 36494 & 631 & 65 & 42 \\
\hline David & 39944 & 1181 & 107 & 94 \\
\hline Emma & 71984 & 3988 & 172 & 165 \\
\hline Félix & 67212 & 3131 & 140 & 233 \\
\hline Gisele & 124008 & 7334 & 207 & 240 \\
\hline Hugo & 101351 & 5300 & 207 & 12 \\
\hline Irene & 12852 & 381 & 16 & \\
\hline
\end{tabular}

Tabla 2. Tabla general del total de impresiones, interacciones, «me gusta» $\mathrm{y}$ «retuits» obtenidos por los tuits con etiqueta tipo \#BorrascaNombre.

\section{ANÁLISIS DEL IMPACTO DE LA OSCILACIÓN DEL ATLÁNTICO NORTE (NAO) EN EL NOMBRAMIENTO DE BORRASCAS POR EL GRUPO SW}

Pese a que el periodo de estudio se ha limitado a tres años, se ha intentado analizar el efecto que puede tener un patrón de teleconexión, como es la NAO, en la frecuencia de nombramiento de borrascas dentro del grupo SW. Se ha limitado el estudio a los meses de invierno, de diciembre a marzo, ya que es el periodo en el que se concentra la entrada de borrascas en las latitudes que nos afectan y en el que parece haber una mayor correlación entre la NAO y los tipos de tiempo de España, al menos en la vertiente atlántica.

Los cambios en los patrones de circulación medios sobre el Atlántico norte asociados a la NAO están acompañados por cambios en la intensidad y el número de borrascas, sus trayectorias y el tiempo meteorológico que llevan aparejado. Durante el invierno, existe una clara trayectoria de las borrascas que conecta las cuencas del Pacífico Norte y Atlántico Norte, con una mayor actividad sobre los océanos. En general, los inviernos con un índice NAO positivo están asociados con un desplazamiento hacia el noreste de la circulación de las borrascas atlánticas, con una mayor actividad de Terranova al norte de Europa y una pequeña disminución de la actividad hacia el sur (Hurrel et al., 2013). Así pues, los periodos de tiempo que teóricamente deberían ser favorables al nombramiento de un mayor número de borrascas por el Grupo SW son aquellos en los que la NAO se encuentra en su fase negativa. 
En la figura 6 se puede observar cómo en las tres últimas temporadas invernales, únicamente el mes de marzo de 2018 se desarrolló dentro de un periodo de NAO negativa.

En este mes, se nombraron tres borrascas, Félix, Gisele y Hugo, y apenas días antes se nombró Emma. Todas ellas fueron nombradas por AEMET o IPMA, ya que llegaron a afectar a latitudes inferiores a $45^{\circ} \mathrm{N}$. Por ello, y a falta de extender el estudio a un periodo más amplio, se puede concluir que existe una clara correlación entre la NAO negativa mensual y la frecuencia de borrascas nombradas por el Grupo SW.

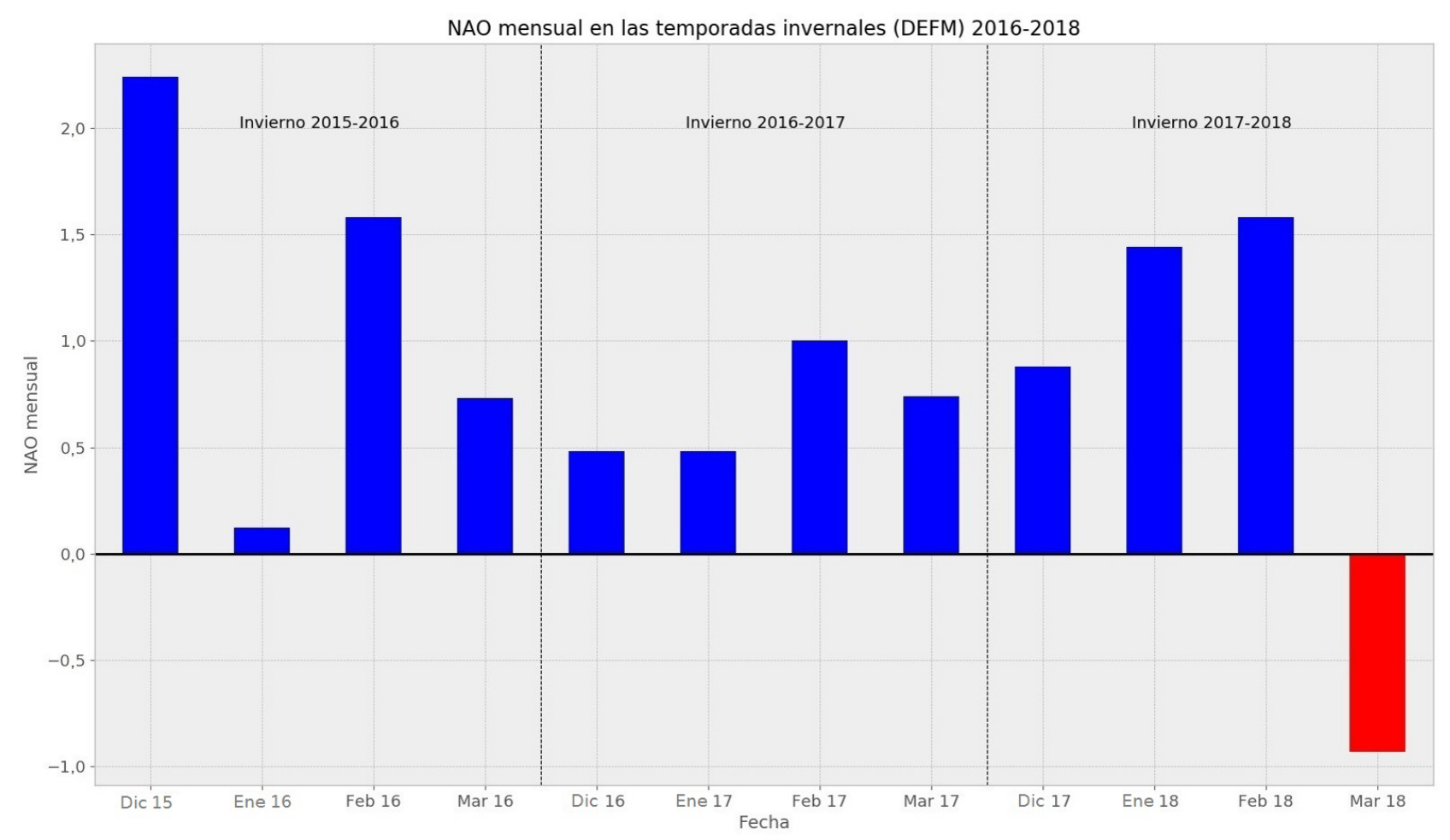

Figura 6. Variación mensual de la NAO durante las tres últimas temporadas invernales (de diciembre a marzo).

\section{NOVEDADES PARA LA TEMPORADA 2018 -2019}

De cara a la temporada 2018-2019 y con el fin de mejorar tanto la coordinación interna como la difusión del nombramiento de borrascas se van a implementar una serie de cambios y mejoras:

a) Mayor coordinación entre los grupos Suroeste y Oeste (intercambio entre todos los Servicios Meteorológicos Nacionales implicados de un formulario de nombramiento normalizado).

b) Nombramiento de las borrascas con gran impacto en el Mediterráneo occidental además de las del Atlántico.

c) Inclusión del nombre de las borrascas nombradas por el Grupo Oeste en los mapas de frentes de AEMET.

d) Creación de un apartado en la web de AEMET para su seguimiento: http://www.aemet.es/es/conocermas/borrascas/2018-2019.

e) Nuevo listado de nombres para el Grupo Suroeste para la temporada 2018-19, que empieza el 1 de octubre. 


\section{CONCLUSIONES}

Las principales conclusiones a las que se ha llegado con este estudio han sido las siguientes:

a) El número de visitas a la web de AEMET aumenta mucho en los episodios en los que se nombran las borrascas, pero también en otras situaciones con nevadas y precipitaciones intensas de gran impacto.

b) En todas las borrascas nombradas que afectaron a España, además de avisos naranjas/rojos de viento, hubo también naranjas/rojos costeros y/o de nevadas.

c) Los tuits con los nombres de las borrascas tienen un gran seguimiento, especialmente el primero de ellos al nombrarse («tuit destacado») y cuando la borrasca afecta especialmente a España, con lo cual se gana seguimiento de la cuenta @ AEMET_Esp ya antes de que entren en vigor los avisos naranjas/rojos emitidos por AEMET.

d) No se puede llegar a una conclusión clara sobre si el nombramiento de borrascas ha hecho aumentar el número de visitas a la web, pero parece que ayuda a la difusión del episodio y sus avisos asociados a través de los medios de comunicación.

e) Hay una clara correlación entre la NAO negativa mensual y la frecuencia de borrascas nombradas por IPMA y AEMET.

f) El nombramiento de borrascas es una oportunidad para intercambiar información entre predictores de diferentes SMHN europeos y mejorar en la coherencia de los avisos transfronterizos.

g) El objetivo final es tender a una lista única a nivel europeo, con una plataforma que permita tanto intercambiar información como comunicar el nombre.

Finalmente, sería muy interesante extender este trabajo fuera del ámbito de difusión de AEMET, analizando el efecto y alcance que tuvo el nombramiento de borrascas en los medios de comunicación españoles.

\section{AGRADECIMIENTOS}

A Pablo Serrano Molinero (becario del Área de información meteorológica y climatológica de AEMET), al Área de Explotación y Gestión de Datos de AEMET y a Alicia García Ortega del Centro Nacional de Predicción de AEMET.

\section{REFERENCIAS}

[1] https://public.wmo.int/en/About-us/FAQs/faqs-tropical-cyclones/tropical-cyclone-naming.

[2] http://www.met.fu-berlin.de/adopt-a-vortex/historie/.

Cusack, E., Paterson, L., Lang, W. y Csekits, C., 2017. WGCEF Task Team on Storm Naming in Europe. The European Forecaster. Newsletter of the WGCEF (N. 22). http://euroforecaster.org/newsletter22/ storm.pdf.

Hurrell, J.W., Kushnir, Y., Ottersen, G. y VisbeCk, M., 2013. An Overview of the North Atlantic Oscillation. In The North Atlantic Oscillation: Climatic Significance and Environmental Impact (eds J. W. Hurrell, Y. Kushnir, G. Ottersen y M. Visbeck). 
